\title{
Distributed Scheduling via Pricing in a Communication Network
}

\author{
Tiina Heikkinen \\ Department of Economics \\ University of Crete, Rethymno \\ 74100 Greece \\ Heikkinen@econ.soc.uoc.gr
}

\begin{abstract}
This paper addresses the issue of pricing-based distributed resource allocation via scheduling in a communication network. Introducing the temporal aspect in the resource allocation problem presents new challenges e.g. in accounting for the durations and deadlines of the resource requests. Dynamic real-time pricing concepts are discussed for the decentralized sharing of network resources. A numerical example illustrates the benefit of congestion based pricing in a dynamic communication network. The quality of service that results from decentralized resource allocation is studied from the point of view of a power-controlled wireless network. The model is based on a dynamic noncooperative game and is related to recent work on centrally optimal (Pareto-optimal) distributed dynamic resource allocation.
\end{abstract}

Technical subject area: Scheduling, Quality of Service, Auctions, Power Management

\section{Introduction}

This paper addresses the issue of efficient dynamic resource allocation via distributed scheduling in a communication network. Examples of networks where resource allocation is distributed in nature include e.g. the Internet and an $a d-$ hoc wireless network where the mobile users are not always directly connected to a base station. Such self-organizing networks necessitate decentralized solutions to resource allocation. Introducing the temporal aspect in the resource allocation problem presents new challenges: it becomes necessary to explicitly account for the specific deadlines and durations of the resource requests made by independent users.

The decentralized resource allocation in a communication network has been recently discussed in terms of an efficient combinatorial auction 12. This paper presents an alternative approach to centrally optimal, i.e. Pareto-optimal decentralized dynamic resource allocation focusing on the allocation of a divisible network resource such as bandwidth in a communication network. Previously, [10] has illustrated how game theory can be used to analyze decentralized resource allocation in congested networks. This paper discusses how dynamic game theory applies in the analysis of the distributed resource allocation in a communication

E. Gregori et al. (Eds.): NETWORKING 2002, LNCS 2345, pp. 850-862 2002.

(C) Springer-Verlag Berlin Heidelberg 2002 
network. Recently, in 2] an analogous model for optimal dynamic decentralized resource allocation is presented; here like in [2] the approach is based on reducing a dynamic game to an auxiliary static game. The application to distributed bandwidth allocation in a communication network presented in this paper can be seen as a special case of the framework in [2].

This work applies a model for distributed dynamic resource allocation (cf. [2]) and focuses on the optimal quality of service $Q o S$ in a distributed dynamic network and the mechanisms through which the optimal $Q o S$ can be reached. Congestion based pricing is shown to constitute an optimal control in a decentralized dynamic network. The $Q o S$ of user $i$ is measured in terms of the share of bandwidth allocated to user $i$. An example of such QoS measure is the signal-to-noise ratio in a power-controlled wireless network, see appendix A. The organization of the paper is as follows. Section 2 introduces the scheduling problem and presents the special case of a static network in 2.1. In 2.2 a general model for dynamic resource allocation is summarized; it is argued that the application to a communication network results as a limiting special case of the model in [2]. Section 3 presents models for dynamic distributed resource allocation in a communication network and discusses the connection of a pricing-based allocation model to auction games. Section 4 discusses optimal dynamic pricing and the connection between the asynchronous static network that appears as a dynamic network and the dynamic network that can depict an asynchronous "static" network (cf. [1]). The dynamic resource allocation problem in a congested communication network is related to the general load sharing problem in a capacitated network.

\section{A Model of the Scheduling Problem}

This paper discusses game theory under incomplete information in a setting where $m$ users share bandwidth in a time-varying network. The general dynamic resource allocation problem can be defined in terms of the following elements:

- a set of $T$ discrete time slots,

- a set $M=\{1, \ldots, m\}$ of $m$ players (users) and a competitive seller (network), each with a concave payoff function $w_{i}, i=1, \ldots, m+1$,

- resource prices $\mathbf{p}=\left(p_{1}, \ldots, p_{T}\right)$ at the different periods.

If the resource request for all users consists of one time slot, the scheduling problem is called single-unit (discrete) scheduling [12]; under multiunit scheduling the users may request multiple slots. In this paper the users request a part of bandwidth each time period. This part may or may not correspond to a single period or slot. Let $x_{i t}$ denote the resource allocated to user $i$ at period $t$. The $i$ th user's duration $D_{i}$ is defined as: $D_{i}=\sum_{t=0}^{T_{i}} J_{t}\left(x_{i t}\right)$, where $J_{t}\left(x_{i t}\right)=1$ if $x_{i t}>0$ and $J\left(x_{i t}\right)=0$ otherwise. User $i$ 's resource request $\left(x_{i t}\right)_{t=1}^{T_{i}}$ is defined by its duration, its deadline $d \leq T_{i}$ and its value $w_{i}$ :

$$
w_{i}=\sum_{t=0}^{T_{i}} b^{t} u_{i t}\left(x_{i t}\right)-c_{i t} .
$$


The discount factor is denoted by $b \in(0,1)$. The $i$ th user gets no value unless the request is satisfied before the deadline $d_{i}$. For this, $c_{i t}=0 \forall t<d_{i}, c_{i d_{i}}=$ $\sum_{t=0}^{d_{i}} b^{t} u_{i t}$ and $c_{i t}=b^{t} u_{i t}$ when $t>d_{i}$.

Definition 2.1. A decentralized scheduling system is defined as a system where each user $i=1, . ., m$ solves $\max E\left[w_{i}\right]$ such that $x_{i t} \leq R_{i t}$, where $E$ denotes the expectation operator and where the resource constraint $R_{i t}$ is given.

Let $h\left(\mathbf{u}_{t}\right)=\sum_{i=1}^{m}\left[u_{i t}-c_{i t}\right]$ denote an aggregating function; a centralized system maximizes the aggregated objective function

$$
\max E\left[\sum_{t=0}^{T} b^{t}\left(\sum_{i=1}^{m} u_{i t}-\frac{c_{i t}}{b^{t}}\right)\right]=\max E\left[\sum_{t=0}^{T} b^{t} h\left(\mathbf{u}_{t}\right)\right]
$$

subject to $\sum_{i=1}^{m} x_{i t} \leq R_{t} \forall t$, where $R_{t}$ denotes the resource constraint at period $t$ (cf. [2] ). The mechanism design problem is to find prices such that the decentralized (noncooperative) solution to the scheduling problem coincides with the centralized (cooperative) solution. The issue of optimal dynamic pricing is taken up in section 4 .

Let $g_{i}$ denote the network link coefficient of user $i$ [13]. Let $\alpha_{i}=\frac{y_{i}}{I_{i}}$ denote the quality of service $Q o S$ of user $i$ in the network, where $y_{i}=g_{i} x_{i}$ denotes the received signal of users $i$ and $I_{i}$ denotes the total interference of $i$. The $Q o S$ $\alpha_{i}$ can be interpreted as the signal-to-noise ratio and it is the argument in $u_{i}$. Let $p$ denote the unit price for $y$. The special static case where $T=1, b=1$, $c_{i t}=0$ and $r_{i}=u_{i}(\alpha)-p y$ has been studied in [6] and is discussed below in 2.1 and 3.3. The connection of this simplified model to distributed dynamic resource allocation is summarized in section 4 .

\subsection{Distributed Resource Allocation in a Static Communication System}

Recently, decentralized resource allocation in has been studied within the framework of general equilibrium theory in [8] and [12 " the market-oriented programming approach") for the Internet and in 6] for a wireless communication network. In a static (one period) wireless network a number of users $m$ need to share the same time slot. Define the quality of service $(Q o S)$ in terms of signalto-noise-ratio $(S N R)$ in a power controlled wireless network 13. Letting the users be the players and the signal-to-noise ratio of a user define the payoff of the user, the $S N R$-model defines an externality game [5].

Definition 2.2. An externality game (EG) is a noncooperative game defined by $m$ players with submodular payoff functions $u_{i}, i=1, . ., m$.

The key restriction on a representative user $i$ 's payoff $S N R u_{i}=\alpha_{i}$ is that it depends on his own strategy, here in terms of received signal power $y_{i}$, and the externality vector $\left(y_{j \neq i}\right)$ through $I=I(\mathbf{y})$. Let $g_{i}$ denote the link fading coefficient for user $i$; thus, define (cf. [136])

$$
u_{i}=\alpha_{i}\left(x_{i}, I(\mathbf{y})\right)=\frac{W g_{i} x_{i}}{I(\mathbf{y})}=\frac{g_{i} x_{i}}{\sum_{j \neq i} g_{j} x_{j}}=\frac{y_{i}}{\sum_{j \neq i} y_{j}}
$$


where $W=1$ denotes the given bandwidth. Here $\alpha_{i}$ is a submodular function i.e. function with decreasing differences: for all $\mathbf{y}, \mathbf{y}^{\prime}$ in the strategy space and $\mathbf{y}>\mathbf{y}^{\prime}$,

$$
\alpha_{i}(\mathbf{y}, I)-\alpha_{i}\left(\mathbf{y}^{\prime}, I\right)=\frac{y_{i}-y_{i}^{\prime}}{\sum_{j \neq i} y_{j}}
$$

is nonincreasing in $I$. Thus, a communication network with limited bandwidth is an example of an externality game motivating externality, i.e. congestion pricing. A non-atomic game is a game with a continuum (large number) of users, in which no single player can affect the other players by changing only her own action. The impact of a single user on the total interference is negligible if the number of users is large. The users as noncooperative players ignore the impact of their usage of resource (transmit power) on the congestion externality, interference, caused to other users.

Recently [6] has discussed two approximately equivalent max-min fair congestion based pricing strategies in an interference limited wireless system:

- QoS pricing consisting of defining a congestion based unit price $p_{\alpha}=p I, p>$ 0 for $Q o S \alpha$ (based on [4]);

- Pricing for received signal power $y$ consisting of a strictly positive unit price $p$ for $y$.

The equivalence is based on considering the first order optimization condition with respect to $y_{i}$ under power-based pricing, given $I_{i}$ (cf. current $C D M A$ ):

$$
\frac{d u_{i}}{d y_{i}}=\frac{1}{I_{i}} u^{\prime}\left(\frac{y}{I_{i}}\right)-p=0,
$$

equivalent to the first order condition under $Q o S$-pricing:

$$
u^{\prime}\left(\alpha_{i}\right)=p I=p_{\alpha} .
$$

Here $I$ denoted interference caused, and when $I \approx I_{i}$ (interference received), the equivalence holds. Based on this, consider only power-based pricing in what follows.

\subsection{A General Model for Distributed Dynamic Resource Allocation}

Sometimes it is assumed for simplicity that $I(\mathbf{y})=\sum_{i=1}^{m} g_{i} x_{i}=\sum_{i=1}^{m} y_{i}$ (cf. [8]). I.e. the utility function of a representative agent $i$ is given by

$$
u_{i t}=\frac{y_{i t}}{\sum_{i=1}^{m} y_{i t}} .
$$

The form in (7) is a special case of the parameterized family of utility functions in [2] (where $\left.c_{i t}=0 \forall i, t\right)$ :

$$
u_{i t}=\frac{y_{i t}}{h(\mathbf{y})^{1-\beta}}
$$


where $\beta \in(0,1)$ and $h$ denotes the aggregating function, assumed to be convex and homogeneous of degree 1 . The aggregating function $h(\mathbf{u})$ for a given time period then satisfies the following [2] equality:

$$
h(\mathbf{u}(\mathbf{y}))=h(\mathbf{y})^{\beta} .
$$

Note that the utility functions in (8) define an externality game.

Proposition 2.3. The utility function (7) for a user in a congested communication network (e.g. interference-limited wireless network or Internet) corresponds to the case of the family of functions (8) when $\beta=0$. The aggregating function $h$ satisfies (9) also in this case.

Proof. Letting $\beta=0$ and $h(\mathbf{y})=\sum_{i=1}^{m} y_{i t}=I(\mathbf{y})$ in (8) yields (7). Also, (9) is satisfied:

$$
h(\mathbf{u}(\mathbf{y}))=\sum_{i=1}^{m} \frac{y_{i t}}{\sum_{i=1}^{m} y_{i t}}=1=\left(\sum_{i=1}^{m} y_{i t}\right)^{0}=h(\mathbf{y})^{\beta} \forall t .
$$

The limiting case when $\beta=0$ is not addressed in [2]. Simplified solutions to the distributed dynamic resource allocation problem corresponding to centrally optimal Nash equilibria are discussed next.

\section{Solution Concepts in a Dynamic Network}

\subsection{Social Nash Equilibria in the Non-cooperative Network Game}

Consider the model in 2.2 for distributed resource allocation. Assume that the users have utility functions $w_{i}$ where $u_{i t}$ s are as in (7). Let $d=T_{i}=T$ be the deadline for all agents. Each user $i$ has initial endowment $R_{i 0}$. At each stage the user should divide his resource into

- $x_{i t}$ for obtaining immediate gain $\alpha_{i t}$ given $g_{i t}$ and the choices of other users

$-z_{i t}=R_{i t}-x_{i t}$ for savings. Let $R_{i t+1}=z_{i t}$ (the "cake-eating" problem in [2]).

The model of resource allocation may be regarded as a non-cooperative dynamic stochastic game 2]. The main solution concept in noncooperative network games (cf. 8]) is a Nash equilibrium [9].

Proposition 3.1. The centrally optimal Nash equilibrium strategy is to use the share of resources depending only on the remaining time periods:

$$
x_{i t}^{*}=R_{i t}\left(\frac{1-b}{1-b^{T-t+1}}\right) .
$$

Proof. Analogous to the proof in [2] for $\beta \in(0,1)$.

I.e. the results in $[2$ hold also when $\beta=0$ in (8), the case corresponding to the utility function model in a congested communication network ( 86$]$ ). Equation (11) indicates that the user becomes more impatient as the deadline $T$ approaches. 
Section 3.2 presents a simplified example for a social Nash equilibrium, an application of the general model above to a distributed network where the users are allowed to decide each period only whether to participate or to opt out. I.e. $x_{i t} \in\{0,1\}$, for all the iterations $t$ for all users $i$, and the comparison is between $\alpha_{i t}=\frac{g_{i t}}{\sum_{j=1}^{m} g_{j t}}$ and $b E\left(\alpha_{i t+1}\right)=b E\left(\frac{g_{i, t+1}}{\sum_{j=1}^{m} g_{j, t+1}}\right) \forall i, \forall t$. The relation between the dynamic model in 3.2 and an analogous model in 3.3 is summarized in section 4 from the point of view of optimal dynamic pricing.

\subsection{A Delay-Limited Solution to Resource Allocation}

The birth-death process of new users entering and users whose deadlines passed leaving make the channel time-varying. Alternatively or simultaneously the link coefficients $\left(g_{i} \mathrm{~s}\right)$ are random variables. In a time-varying channel with a time cost, the participation externality game can by described as follows.

\section{A Centralized Solution to Dynamic Resource Allocation}

Let $y_{i}=g_{i} x_{i}$ denote the received signal, where $g_{i}=(\mathbf{G})_{i i}$ is the fixed link coefficient and $x_{i}$ denotes the power allocation of $i$. Let $f_{i j}=(\mathbf{F})_{i j}=g_{j}$ be the coefficient of interference received by user $i$ from $j$ [13]. Let $\mathbf{y}=\left(y_{i 0}, . ., y_{i T}\right)_{i=1}^{m}$ and let the duration be equal across users $T_{i}=T \forall i$. The dynamic resource allocation problem can be stated as, when taking into account the delay costs formalized by $b \in(0,1)$ and an additive deadline cost $c_{t}$, equal across users (cf. $[5])$,

$$
\max _{\mathbf{y}} E \sum_{t=0}^{T} b^{t} \alpha_{t} \text { s.t. } \quad \mathbf{F}_{t} \mathbf{x}_{t} \alpha_{t}=\mathbf{G}_{t} \mathbf{x}_{t} .
$$

The $Q o S$ of $i$ is measured by $\alpha_{i}=\frac{(\mathbf{G x})_{i}}{(\mathbf{F x})_{i}}$. If $g_{i}=g=1$ is fixed, $\alpha_{t}=$ $\frac{1}{m_{t}} \forall t$ (Appendix A); only the number of users in the network varies over time. Therefore, letting $x_{i t}=y_{i t} \in\{0,1\} \forall i, \forall t$, the scheduling problem becomes a load-balancing problem over time when the network coefficients are fixed. In general, the network coefficients are random variables. Let e denote the $m$-vector of ones. In what follows assume the randomness in $\alpha$ is also due to time-varying fading conditions as captured by the random link coefficient $g$, initially letting $\mathbf{x}=\mathbf{e}: \alpha_{i}=\frac{g_{i}}{\sum_{i=1}^{m} g_{i}} \forall i$ where also $m$ can be a random variable.

Let there be two state variables, $\alpha$ and $s: s=0$ if $\alpha$ for the representative user (omitting user index) is currently not in the system and $s=1$ if the user participates in the network. If the randomly chosen user accepts current $\alpha_{t}$ he stays in the system for period $t$, is absent period $t+1$ and makes a new decision at $t+2$. The Bellman equations for $v(\alpha, s), s=0,1$ are, assuming there is in addition to the deadline delay cost defined by $c$ a proportional delay cost component $b \in(0,1)$ when deferring the choice of the resource usage to the next period $t+1$

$$
\begin{aligned}
& v(\alpha, 0)=\max \{\alpha+b E v(\alpha, 1), b E v(y, 0)-c\} \\
& v(\alpha, 1)=\max \{b E v(y, 0)\} .
\end{aligned}
$$


Define the threshold $\bar{\alpha}$ as in optimal stopping problems by

$$
\bar{\alpha}_{t}+b E v\left(\bar{\alpha}_{t}, 1\right)=b E v(y, 0)-c_{t},
$$

where $\bar{\alpha}$ is the threshold $Q_{o S}$. Note that $\frac{\sum_{i=1}^{m} E v\left(\alpha_{i}, s\right)}{m}=E v(\alpha, s)$ assuming the $g_{i}$ s are independent and identically distributed random variables. Let this model define the expected value of $Q o S$ as [5]:

$$
E v(\alpha, 0)=\frac{\bar{\alpha}_{t}(1+b)}{b(1-b)}+\frac{c_{t}}{b(1-b)} .
$$

The optimal threshold $\bar{\alpha}$ can be characterized as: $\bar{\alpha}_{t}=\frac{E(\alpha)-\int_{0}^{\bar{\alpha}} \alpha f(\alpha) d \alpha-\frac{c_{t}}{b}}{\frac{1}{b}+(1-F(\bar{\alpha}))}$ where $F$ is the cumulative density function of $\alpha$ and $f$ denotes the distribution function of $\alpha=\left(g_{i}\right) /\left(\sum_{i} g_{i}\right)$.

\section{Noncooperative Equilibrium in Dynamic Resource Sharing}

It can be shown that the centralized model summarized by equations (3.2) is equivalent to a simplified version of the noncooperative model in [2] with Paretooptimal outcomes, where the corresponding utility function of user $i$ in a one-shot auxiliary game for strategy profile $\mathbf{x} \in\{0,1\}^{m}$ is:

$$
r_{i}=\frac{x_{i}}{I(\mathbf{x})}+b \frac{\left(1-x_{i}\right)}{I(\mathbf{e}-\mathbf{x})}
$$

where $R_{i t}=1=e_{i} \forall i$ (cf. 3.1). The first order condition (f.o.c) of user $i$ is to let $I\left((\mathbf{x})^{*}\right)+x_{i}^{*} \frac{d I(\mathbf{x})^{*}}{d x_{i}}=b\left(I\left(\mathbf{e}-\mathbf{x}^{*}\right)+\left(e_{i}-x_{i}^{*}\right) \frac{d I\left(\left(\mathbf{e}-\mathbf{x}^{*}\right)\right.}{d x_{i}}\right)$. By the homogeneity of $I, \sum_{i=1}^{m} x_{i} \frac{d I(\mathbf{x})}{d x_{i}}=(\beta-1) I(\mathbf{x})=-I(\mathbf{x})$. When summing the f.o.c.s this implies that at the deterministic Nash equilibrium $I\left(\mathbf{x}_{t}^{*}\right)=b I\left(\mathbf{x}_{t+1}^{*}\right)$ equivalent to: $\alpha_{t}=$ $b \alpha_{t+1}$, as in the load-balancing Pareto-optimal solution. The corresponding $\bar{\alpha}$ is defined by $m_{t}$ such that: $\bar{\alpha}=\frac{1}{m_{t}}=\frac{b}{m-m_{t}}$.

The above centralized formulation also directly captures a noncooperative model, due to the symmetry across users in terms of the parameters $b, c$ and $u_{i}$ (identity). The noncooperative users simultaneously or asynchronously pick a strategy $x_{i t}$ from $A=\{0,1\}$ (or, if only $m$ varies, $y_{i t} \in A$ ) each period $t$ when the $Q o S$ was rejected at $t-1$ or accepted at $t-2$. The centralized case above assumed that the distribution $f$ of $\alpha$ is stationary. This need not be the case in a time-varying network. However, assume that the environment becomes stationary after some finite time. Then, as argued in [5], a simple (participation) $E G$ such as defined above (by $\operatorname{card}(A)=2$ ) is $D$-solvable which implies that users in the linear utility model will converge to a unique Nash-equilibrium even in noisy distributed asynchronous settings. The argument is as follows. Given the ultimately stationary distribution of the number of users, the strategy choices of the other users in terms of $\alpha$ and $E v(\alpha)$ appear as parameters in the participation decision.

Note that formally the maximization of $\alpha$ is equivalent to the maximization of $b \alpha-p y$, letting utility parameter $b$ satisfy: $b=1+p_{\alpha}$, where $p_{\alpha}=I p$. By tuning the price the $Q_{o S}$ in the network can be maintained at the desired level. 


\subsection{The "Static" Network Can Be Seen as a Dynamic Game}

The iterative solution of the static distributed resource allocation has connections to a dynamic game.

Definition 3.2. A game is said to be dynamic if at least one of the players has more than one information set [1].

An information set is a concept for games in extensive (tree) form [1]: the same strategy set is available at each node in the same information set, where the nodes correspond to possible events; no node follows another node in the same information set.

Consider the following iterative solution of a resource (bandwidth=1) sharing game in a wireless system where $T=1[\underline{6}$.

Definition 3.3. Let $A_{i}=\{0,1\}$ denote the strategy set [1] of user $i=1, . ., m$. An asynchronous greedy algorithm for the game defined by the payoff functions

$$
r_{i t}=u_{i t}\left(\alpha_{i t}\right)-p y_{i t}, i=1, . ., m,
$$

where $\alpha_{i t}=\frac{y_{i t}}{I_{i t}+e}, I_{i t}=\sum_{j \neq i} y_{j t}, e=\alpha n=0.0001$ (see Appendix A) and the $u_{i t} s$ are given in (7), consists of iteratively finding, for $i=1, \ldots, m$,

$$
y_{i t}^{*}=\arg \max _{y_{i t} \in A_{i}} u_{i}\left(\frac{y_{i t}}{I_{i t}+e}\right)-p y_{i t}
$$

The above greedy algorithm defines a solution to bandwidth-scheduling under a price penalty when $R_{i t}=1 \forall i$ (i.e. the resource constraints are due to $A_{i}=$ $\{0,1\} \forall i \forall t)$. The users opting in at period $t$ set $y_{i}^{t}=1$. The corresponding $Q o S$ price at period $t$ is $p_{\alpha}=p m_{t}$, where $m_{t}$ denotes the number of users present in the network at period $t$.

Consider the tree representation of the greedy algorithm. At period $t=1$, a user $i$ is picked randomly to update its resource demand given $I_{1}$ (given the initial values of the other users choices). The corresponding node in the game tree is the root of the tree. The following period $t=2$ another user from the set $M$ is chosen randomly to update its demand, given $I_{2}$. Iterating the tree construction yields a binary tree where each user faces the information set consisting of all nodes (events): given the total interference $I_{t}$, the user is unable to determine the decomposition of $I_{t}$, i.e. which other users are present.

The sequential solution of the originally static game thus yields a dynamic game where each user $i$ will make a decision (reach an information set) $k$ times where $k$ is the time per user that the greedy algorithm takes before converging to a Nash equilibrium. By the concavity of the payoff functions such an equilibrium exists. The reason for the "static" game to appear as a dynamic game is that each player acts more than once and hence time plays a role (cf. [1]). Figure 1 depicts the total time required for the greedy algorithm in the special case of linear utility model where $u_{i}\left(\alpha_{i}\right)=b_{i} \alpha_{i}=i \alpha_{i} \forall i, m=20, p=1$. Even when adding the dominated strategies $([9])\left\{0.5, \ldots, \frac{1}{20}\right\}$ the greedy algorithm converges to the Nash equilibrium in less than five iterations per user. Only the users with high utility participate in the network, i.e. users $i=11, \ldots, 20$, yielding the sum of utilities 17.22 at the Nash equilibrium (which can be easily verified). 
In the absence of a price $p=0$ the sum of utilities is lower (11.05). At $p=19$, the sum of utilities is at highest 39 . But for users $i=9, \ldots, 18$ this equilibrium yield less than the equilibrium under $p=1$ and hence does not constitute a Pareto-improvement from the Nash equilibrium at $p=1$.

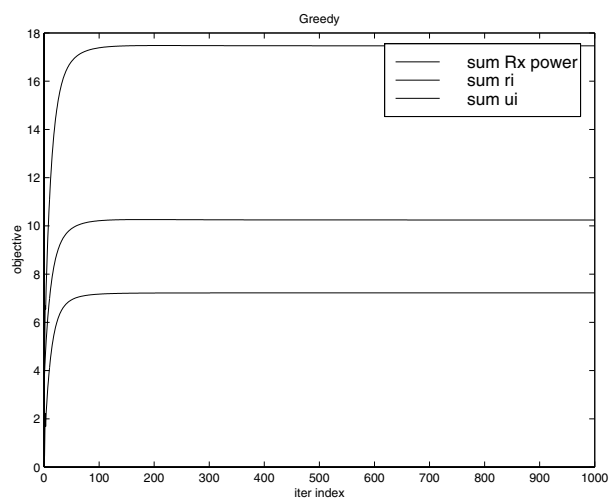

Fig. 1. Greedy Algorithm: Sum of Utilities (topmost curve), Received Powers (middle curve) and Net Utilities (lowest curve) for $m=20, p=1, u_{i}=i \alpha_{i}, A_{i}=$ $\left\{1,0.5, \ldots, \frac{1}{20}, 0\right\}, i=1, . ., m, e=0.0001$.

\section{Dominance Solvable Network Games}

In the linear utility example in section 2 where $u_{i}\left(\alpha_{i}\right)=i \alpha_{i}$, the middle strategies are strictly dominated 9 . In a linear model it suffices to consider participation games where $A_{i}=\{0,1\}, i=1, \ldots, m$. Participation games constitute an important class of externality games $E G$ s. The main result for participation $E G$ s is related to their dominance- $D$-solvability [5].

Definition 3.4. Let $u_{i}=\alpha_{i}$. For a given player $i$, strategy $\mathbf{y}$ dominates strategy $\mathbf{y}^{\prime}$ relative to strategy set $A$ iff $\alpha_{i}(\mathbf{y}, I)>\alpha_{i}\left(\mathbf{y}^{\prime}, I\right)$ for given $I$. The set of strategies which are not dominated are denoted by $D_{i}(A)$.

Define $D^{k}(A)=D\left(D^{k-1}(A)\right)$ with $D^{1}=D(A)$. Note that $D^{\infty}=\lim _{k \rightarrow \infty} D^{k}$ is well defined due to the monotonicity of $D$.

Definition 3.5. A game is D-solvable if card $\left(D^{\infty}\right)=1$ for all $i$.

$D$-solvability (solvability by dominated strategies) requires that given only partial information of the opponent's strategies as captured by $I_{i t}$, the player still can eliminate dominated strategies.

Proposition 3.6. Let $A_{i}=\left\{0, \frac{1}{2}, . ., \frac{1}{m}, 1\right\} \forall i$. The received power allocation game defined by $m$ payoff functions $r_{i}=\alpha_{i}-$ py $y_{i}$, where $\alpha_{i}=\frac{b_{i} y_{i}}{I_{i}+e}$ where $b_{i}=i$ is $D$ - solvable. 
Proof. Given $I_{i t}$, $e$ and $p$, either $\frac{b_{i}}{I_{i t}+e}-p>0$ implying $y_{i t}^{*}=1$ or $\frac{b_{i}}{I_{i t}+e}-p \leq 0$ implying $y_{i t}^{*}=0$. I.e. for any $t$ and $I_{i t}$ one of the $y_{i t}^{*}=1$ or $y_{i t}^{*}=0$ strategies is better than the other strategies and $D^{\infty} \subseteq\{0,1\} \forall i$. For all $i=1, . ., i_{t h} y_{i}^{*}=1$ and $y_{i}^{*}=0$ for $i<i_{t h}$ where the threshold user $i_{t h}$ is defined as the upper integer part of the solution $i_{t h}$ to $\frac{i_{t h}}{m-i_{t h}-1+e}=p$ :

$$
i_{t h}=\left\lceil\frac{p(m-1+e)}{1+p}\right\rceil .
$$

In the example the greedy algorithm converges to a unique Nash equilibrium. In general, an equilibrium exists in a noncooperative game assuming that the utility functions are concave 9 .

Proposition 3.7. A Nash equilibrium in the resource allocation game defined by the utility functions (16) is Pareto-optimal.

Proof. Consider a given Nash equilibrium allocation $\mathbf{y}_{t}$. The utility of the $i$ th participating agent is $r_{i t}=\frac{b_{i} y_{i t}}{\sum_{j \neq i} y_{j t}+e}-p y_{i t} \geq 0$. Given $b_{i}$ and $p, r_{i t}$ can not be increased by increasing $y_{i t}$ without decreasing $r_{j t}=\frac{b_{j} y_{j t}}{\sum_{k \neq i, j} y_{k t}+e}-p y_{j t} \forall j \neq i$.

\section{A Relation to Auctions}

An alternative solution concept for a dynamic resource sharing game is to consider specific auction protocols. Recently, in [12 an ascending auction protocol for the general discrete resource allocation problem is presented. There is a connection between the auction approach and the linear utility model under a given price $p$. By specifying his utility function $u_{i}$ user $i$ makes an implicit bid consisting of the amount that he is willing to pay at maximum for one unit of transmission. When $I=\sum_{i=1}^{m} y_{i}, b_{i}=i$ and $y_{i} \in\{0,1\} \forall i$, the optimal price inducing at most one user to be present at any time period $t$ under the greedy algorithm is $p_{t}^{*}=\max _{i} i \in\left\{1, . ., m_{t}\right\}=m_{t}$.

\section{Optimal Dynamic Pricing and the Connection between a "Static" Network and a "Dynamic" Network}

A dynamic network as described in section 3.2 above explicitly defines the resource usage problem for a representative user for $T$ time periods. The asynchronous resource sharing game in section 3.3 is concerned with the iterative solution for a "static" resource sharing problem. However, there is a direct relation between the two models.

Under "fictitious play" [3], the users behave as if they think they are facing a stationary (but unknown) distribution of opponent's strategies. The model in section 3.2, where in general the number of users $m$ is a random variable, captures this idea: each period the representative user behaves so as to maximize the expected payoff. In what follows let $0 \leq \beta=\frac{b(1-b)}{1+b}<1$ and for now assume 
that the additive delay cost is zero by letting $c=0$. The user participates in the network at period $t$ letting $y_{t}=1$ if

$$
\alpha_{t} \geq \frac{b(1-b)}{1+b} E v(\alpha, 0)=\beta E v(\alpha, 0)=\bar{\alpha} .
$$

In the "static" linear utility model in section 3.3 the utility of user $i$ at period $t$ was defined as $r_{i t}=i \alpha_{i t}-p y_{t}=u_{i t}\left(\alpha_{i t}\right)-p y_{i t}$. Here consider a symmetric version of this linear model and let $u_{i t}\left(\alpha_{i t}\right)=b^{\prime}\left(\alpha_{i t}\right)$. In the symmetric linear model the representative user participates in the network at period $t$ letting $y_{t}=1$ if

$$
\alpha_{t} \geq \frac{1}{b^{\prime}} p
$$

The decision rules in (19) and (20) coincide if

$$
\beta=\frac{1}{b^{\prime}}
$$

and the price reflects the expected value of $Q \circ S$

$$
p=E v(\alpha, 0)
$$

In a symmetric dynamic Nash equilibrium the users can find the optimal strategy fast applying the greedy algorithm under optimal pricing: $p=E v(\alpha)$.

The relation (21) can be interpreted as follows. The representative user in the linear utility model is more demanding if $b^{\prime}$ is relatively high; this is analogous to a low value of $\beta$ in a temporal allocation model: the greedier user is also the more impatient.

Above letting $c_{t}=0 \forall t$ reflects infinite deadline for simplicity. In the presence of a deadline cost, i.e. when $c_{i d}>0$,

$$
\bar{\alpha}_{d_{i}-1}=\max \left\{E v(\alpha, 0) \frac{b(1-b)}{1+b}-\frac{c_{i d}}{1+b}, 0\right\}<\bar{\alpha} .
$$

I.e. when the deadline period $d_{i}$ is ahead, the acceptance threshold will be lower. Analogously to (22) and (23), the price before deadline becomes

$$
p_{d_{i}-1}=\max \left\{E v(\alpha, 0)-\frac{c_{i d}}{b(1-b)}, 0\right\}
$$

Assuming $p=E v(\alpha, 0)-\frac{c_{d}}{b(1-b)}$ as in (24) at period before deadline $d-1$, and $p=E v(\alpha, 0)$ otherwise, relates the optimal ( $Q o S$-price equivalent) price $p$ to the expected value of $Q o S$ (at state 0) in a symmetric network. For the user to participate, the price in terms of opportunity cost must not be greater than the benefit from participation. In an asymmetric network the different users may have different deadlines corresponding to different $\bar{\alpha}_{i}$ s and the users $i=1, \ldots, m$ iterate the following Bellman equations:

$$
\max _{y_{i t} \in\{0,1\}}\left\{0, b_{i} \alpha_{i t}\left(y_{i t}\right)-\bar{\alpha}_{i t}\right\}, t=0, . ., T_{i} .
$$


The corresponding greedy algorithm successively eliminates dominated strategies in the dominance solvable game (cf. Proposition (3.6)) for users $i=1, \ldots, m$

$$
\max _{y_{i t} \in(0,1)} b_{i} \alpha_{i t}\left(y_{i t}\right)-\bar{\alpha}_{i t} y_{i t}
$$

where $\alpha_{i t}=\frac{y_{i t}}{I_{i t}}=\frac{y_{i t}}{m_{i t}}$. The price $p_{i t}$ is fixed at $p=E v(\alpha)$ until the deadline period $t=d_{i}-1$ of $i$ is reached at which point the price becomes zero if $p_{d_{i}-1}=0$ in (24). Consider the numerical example for the greedy algorithm in section 3.3 where the Nash equilibrium is found in less than five iterations per user such that users $i=1, . ., 9$ converge to setting $y_{i}^{*}=0$. However, suppose the deadline $d_{i}$ for these users is after period 5, e.g. period 6 whereas the deadline for the users $i=10, . ., 20$ with duration five is at period 5 after which they will disappear from the system. Then, the optimal dynamic equilibrium allocation is given by $y_{i t}=1, i=10, . ., 20, t=1, . ., 5$ and $y_{i t}=1$ for $i=1, . ., 9$ at $t=6$ and zero otherwise. Since the users update their strategies in a random order, this equilibrium is an approximately fair efficient solution to dynamic resource allocation.

The socially optimal Nash equilibrium in the network externality game as defined by utility functions in (8) can be rationalized by an implicit congestion based price for $Q o S$, equivalent to a unit price $p$ on $y$ a argued in 2.1. A similar observation is pointed out in 22] if we regard the impact of the externality $h$ on the individual utility as a resource price, then the individual utilities should be proportional to $h^{\beta-1}$. The network externalities are internalized when the payoff functions are as in (8). Likewise, if the $Q o S$ requests are feasible, optimal power control can be distributed [6].

\section{Conclusion}

The focus in this paper has been on centrally optimal distributed scheduling in a communication network with a divisible resource, bandwidth. In some applications the acceptable $Q o S$ level implies that only a single user can be allocated to a given time slot; in the framework of the current paper there is a price level (cf. recent related work [12]) that corresponds to allowing only one user at a time to be present.

An asynchronous resource sharing game over time defines a dynamic game. The scheduling problem adds user-specific durations and deadlines. The uncertainty under dynamic resource allocation comes from the time-varying load (number of users) in the network. An alternative interpretation is to consider time-varying link coefficients.

\section{Appendix A}

The centralized uplink power control problem [13] in a single-cell network can be summarized as follows. Let $\alpha$ denote the $Q o S$ requirement in terms of the signal-to-noise ratio. Let $x_{i}$ denote transmit power allocation of user $i$, let $g_{i j}$ denote the fading coefficient between mobile $i$ and base $j$ and let $n$ be the 
additive Gaussian noise. The power control problem [13] is to find $\mathbf{x}$ such that $\mathbf{G} \mathbf{x}=\alpha(\mathbf{F} \mathbf{x}+\mathbf{n}), \quad \sum_{i} x_{i} \leq R$, where $\mathbf{F}$ denotes the interference (externality) matrix and $R$ denotes the resource constraint. When $n=0$, a solution is to let $x_{i}=\frac{1}{g_{i}}$ and $\alpha_{i}=\frac{g_{i} x_{i}}{\sum_{j \neq i} g_{j} x_{j}}=\alpha=\frac{1}{m-1} \forall i$, where $m$ denotes the number of users.

\section{References}

1. T. Basar and G. Olsder: Dynamic Noncooperative Game Theory. Academic Press (1995)

2. V. Domansky and V. Kreps: Social Equilibria for Competitive Resource Allocation Models. proc. Constructing and Applying Objective Functions, Springer Lecture Notes in Economics and Mathematical Systems (2002)

3. D. Fudenberg and D. Levine: The Theory of Learning in Games. The MIT Press (1999)

4. T. Heikkinen: Dynamic Pricing of a Multimedia Network. proc. CISS, Princeton (1998)

5. T. Heikkinen: On Learning and Quality-of-Service in a Wireless Network. proc. Networking, Paris, France (2000)

6. T. Heikkinen: On Congestion Pricing in a Wireless Network. Wireless Networks, forthcoming

7. H. Ji and H. Cing-Yao: Noncooperative Uplink Power Control in Cellular Radio Systems. Wireless Networks 4 (1998)

8. F. Kelly and R. Gibbens: Resource Pricing and the Evolution of Congestion Control. http://www.statslab.cam.ac.uk/ frank/evol.html

9. R. Myerson: Game Theory. Harvard University Press (1991)

10. S. Shenker: Making Greed Work in Networks. IEEE Tr. on Networking 3(6) (1995)

11. N. Shroff, M. Xiao and E. Chong: Utility based power control in cellular radio systems. Proc. Infocom, Anchorage (2001)

12. M. Wellman, W. Walsh, P. Wurman and J. Mac-Kie-Mason: Auction Protocols for Decentralized Scheduling. Games and Economic Behaviour, forthcoming

13. Zander, J.: Performance of Optimum Transmitter Power Control in Cellular Radio Systems. IEEE Transactions on Vehicular Technology (1992) vol. 41, no. 1, Feb. 